\title{
COLORFUL SIMPLICIAL DEPTH, MINKOWSKI SUMS, AND GENERALIZED GALE TRANSFORMS
}

\author{
KARIM ADIPRASITO, PHILIP BRINKMANN, ARNAU PADROL, PAVEL PATÁK, \\ ZUZANA PATÁKOVÁ, AND RAMAN SANYAL
}

\begin{abstract}
The colorful simplicial depth of a collection of $d+1$ finite sets of points in Euclidean $d$-space is the number of choices of a point from each set such that the origin is contained in their convex hull. We use methods from combinatorial topology to prove a tight upper bound on the colorful simplicial depth. This implies a conjecture of Deza et al. (2006). Furthermore, we introduce colorful Gale transforms as a bridge between colorful configurations and Minkowski sums. Our colorful upper bound then yields a tight upper bound on the number of totally mixed facets of certain Minkowski sums of simplices. This resolves a conjecture of Burton (2003) in the theory of normal surfaces.
\end{abstract}

\section{INTRODUCTION}

The classical Carathéodory theorem states that for any point $p$ in the convex hull of a finite set $V \subset \mathbb{R}^{d}$, there is a subset $S \subseteq V$ of at most $d+1$ points with $p \in \operatorname{conv}(S)$. Of course, this is is invariant under translation and for all practical purposes one can always assume $p=0$. In 1982, Imre Bárány [3] gave a beautiful colored version of this result.

Theorem 1.1 (Colorful Carathéodory Theorem). Let $C_{0}, \ldots, C_{d} \subset \mathbb{R}^{d}$ be finite sets such that 0 is in the relative interior of $\operatorname{conv}\left(C_{i}\right)$ for all $0 \leq i \leq d$. Then there is some $S \subseteq C_{0} \cup \cdots \cup C_{d}$ with $\left|S \cap C_{i}\right|=1$ for all $i$ such that $0 \in \operatorname{conv}(S)$.

The sets $C_{i}$ are thought of as color classes and $C=\left\{C_{0}, \ldots, C_{d}\right\}$ is called a colorful configuration. We call $C$ centered if $0 \in \operatorname{relint} \operatorname{conv}\left(C_{i}\right)$ for all $0 \leq i \leq d$. The assumption that $C$ is centered can be considerably weakened (see, for example, $[2,3,13,20]$ ) but in this paper we will only consider centered configurations; see Question 1. We call a subset $S \subseteq \bigcup_{i} C_{i}$ a colorful simplex if $\left|S \cap C_{i}\right| \leq 1$ for all $i$ and we call the simplex $S$ hitting if $\operatorname{dim} S=|S|-1=d$ and $0 \in \operatorname{conv}(S)$.

Inspired by the simplicial depth introduced in [15], Deza, Huang, Stephen, Terlaky [7] introduced the colorful simplicial depth, $\operatorname{depth}(C)$, of a colorful configuration $C$ as the number of hitting simplices of $C$. In particular, they initiated a systematic study of the extremal values of $\operatorname{depth}(C)$ as $C$ ranges over all colorful configurations in $\mathbb{R}^{d}$. The Colorful Carathéodory Theorem asserts that for any centered configuration, there is at least one hitting simplex. For lower bounds on the colorful simplicial depth, Deza et al. confine themselves to the case of colorful configurations in $\mathbb{R}^{d}$ such that the core $\bigcap_{i} \operatorname{conv}\left(C_{i}\right)$ is of full-dimension $d$ and contains

Date: September 18, 2018.

2010 Mathematics Subject Classification. 52C45, 52A35, 05E45, 52Bxx, 52B35.

Key words and phrases. Colorful simplicial depth, Colorful point configuration, Minkowski sum of polytopes, Gale duality, Cayley trick. 
the origin in its interior. In particular, they conjectured that if $\left|C_{i}\right|=d+1$, then

$$
1+d^{2} \leq \operatorname{depth}(C) .
$$

The initial lower bound of $2 d$ from [7] was improved in a series of papers [4, 8, 9, 26] culminating in the resolution of the conjectured lower bound by Sarrabezolles [24]. In [7] also a conjectured upper bound was proposed.

Conjecture 1 ([7, Conj. 4.4.]). Let $C=\left\{C_{0}, \ldots, C_{d}\right\}$ be a centered colorful configuration in $\mathbb{R}^{d}$ with $\left|C_{i}\right|=d+1$ for all $0 \leq i \leq d$ and 0 in the interior of the core. Then

$$
\operatorname{depth}(C) \leq 1+d^{d+1} .
$$

1.1. Upper Bounds on colorful simplicial depth. The first goal of this paper is to prove this conjecture in the following stronger form. We say that a colorful configuration $C$ in $\mathbb{R}^{d}$ is in relative general position if no colorful simplex $S$ of $C$ of dimension $d-1$ contains the origin in its convex hull.

Theorem 1.2. Let $C=\left\{C_{0}, \ldots, C_{d}\right\}$ be a centered colorful configuration in relative general position in $\mathbb{R}^{d}$ with $\left|C_{i}\right| \geq 2$ for all $0 \leq i \leq d$. Then

$$
\operatorname{depth}(C) \leq 1+\prod_{i=0}^{d}\left(\left|C_{i}\right|-1\right) .
$$

The relative general position assumption is natural as otherwise for a colorful configuration with $C_{1}=\{0\}$, we would have the trivial upper bound of $\prod_{i}\left|C_{i}\right|$. In general, even if the origin is contained in the interior of the full-dimensional core, adding several copies of 0 to the color classes produces configurations with a much larger colorful simplicial depth than the expected bound. This behavior can happen even if all classes avoid the origin; for example, by adding several copies of a point $p$ to $C_{0}$ and several of $-p$ to $C_{1}$. To avoid these artifacts that arise from non-generic configurations, one could alternatively require hitting simplices to contain the origin in its interior. The upper bound is then also attained at colorful configurations in relative general position and our result applies. The requirement that $\left|C_{i}\right| \geq 2$ is subsumed by centered and relative general position and is therefore redundant.

Let us first note that the bound of Theorem 1.2 is tight.

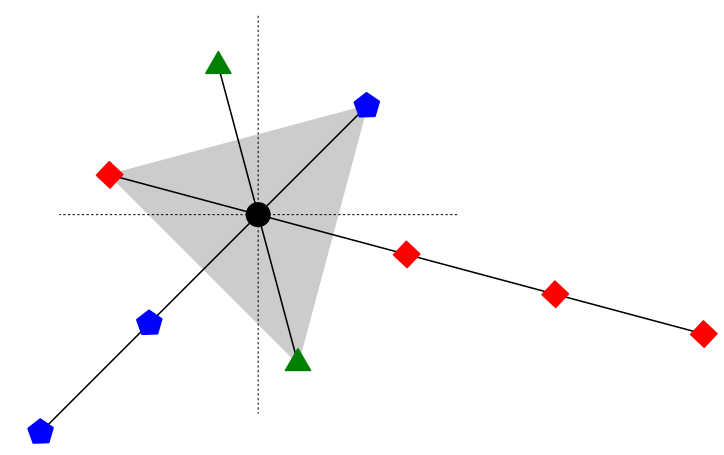

Figure 1. Configuration from Example 1.3 for $\mathbf{n}=(2,3,4)$. 
Example 1.3. Let $v_{0}, \ldots, v_{d} \in \mathbb{R}^{d}$ be the vertices of a simplex containing the origin in its interior and for $n_{0}, \ldots, n_{d} \geq 2$ define

$$
C_{i}:=\left\{v_{i},-v_{i},-2 v_{i}, \ldots,-\left(n_{i}-1\right) v_{i}\right\}
$$

for $0 \leq i \leq d$. Then $C=\left\{C_{0}, \ldots, C_{d}\right\}$ is a centered colorful configuration. The vertices of any colorful $d$-simplex $S$ of $C$ are minimally affinely dependent and hence the coefficients of this dependence are unique up to scaling. It follows that the coefficients are all of the same sign if and only if $S=\left\{v_{0}, \ldots, v_{d}\right\}$ or $S=\left\{-\alpha_{0} v_{0}, \ldots,-\alpha_{d} v_{d}\right\}$ for some $1 \leq \alpha_{i}<n_{i}$. This yields exactly $1+\left(n_{0}-1\right) \cdots\left(n_{d}-1\right)$ hitting simplices.

We will prove Theorem 1.2 in Section 2 by means of combinatorial topology. More precisely, we show that for centered colorful configurations in relative general position, the number of hitting simplices is related to the reduced Betti numbers of an associated simplicial complex, the avoiding complex. In particular, Theorem 1.2 follows from a result that shows that a certain Betti number is independent of the configuration. To show this, we introduce the notion of flips between colorful configurations, and we prove our result by 'flipping' any configuration to the configuration given in Example 1.3.

1.2. Colorful Gale transforms of Minkowski sums. The second goal of this paper is to highlight a connection between colorful configurations and faces of Minkowski sums. The Minkowski sum of convex polytopes $P_{0}, \ldots, P_{s} \subset \mathbb{R}^{d}$ is the polytope

$$
P=P_{0}+\cdots+P_{s}=\left\{p_{0}+\cdots+p_{s}: p_{i} \in P_{i} \text { for } 0 \leq i \leq s\right\}
$$

and this operation is key to many deep results in many areas, notably convex geometry [25] and computational commutative algebra (eg. [10]). The combinatorial complexity of Minkowski sums of polytopes has been subject of several studies [1, 18, 22].

Using Gale transforms and Cayley embeddings, we define in Section 3 colorful Gale transforms associated to a collection $P_{0}, \ldots, P_{s}$ that, similar to ordinary Gale transforms, capture the facial structure of Minkowski sums in the combinatorics of colorful configurations.

A particularly interesting case is that of Minkowski sums of simplices. A face $F$ of the Minkowski sum $P$ is of the form $F=F_{0}+\cdots+F_{s}$ for faces $F_{i} \subseteq P_{i}$. A face is mixed if $\operatorname{dim} F_{i}>0$ for all $i$ and totally mixed if each $F_{i} \subset P_{i}$ is an inclusion-maximal face, that is, $F_{i}$ is a facet of $P_{i}$. If each $P_{i}$ is a simplex, then we show in Lemma 3.7 that totally mixed facets are exactly the hitting simplices containing 0 in their interior of the associated colorful Gale transform. In particular, this allows us to prove the following.

Theorem 1.4. For $n_{0}, \ldots, n_{s} \geq 1$, set $n=n_{0}+\cdots+n_{s}$ and let $P_{i} \subset \mathbb{R}^{n-s-1}$ for $0 \leq i \leq s$ be $n_{i}$-dimensional simplices whose Minkowski sum is full-dimensional. Then the number of totally mixed facets of $P_{0}+\cdots+P_{s}$ is at most

$$
1+n_{0} n_{1} \cdots n_{s}
$$

1.3. A motivation from normal surface theory. This dictionary between Minkowski sums and colorful configurations allows us to resolve a conjecture of Ben Burton [6] about the complexity of projective edge weight solution spaces in normal surface theory. We only describe the geometric formulation of the problem and refer to [6, Ch. 5] for the topological connections and implications. Using the terminology of [6], a $d$-fan $\mathcal{F}$ is the collection of three polyhedral cones $L_{1}, L_{2}, L_{3} \subset \mathbb{R}^{d}$ such that $L_{i}$ is linearly isomorphic to $\mathbb{R}^{d-2} \times \mathbb{R}_{\geq 0}$, i.e, each $L_{i}$ is a halfplane, and such that $L_{i} \cap L_{j}=L_{1} \cap L_{2} \cap L_{3} \cong \mathbb{R}^{d-2}$ for all $i \neq j$. This is called the axis of 
the fan, the cones $L_{i}$ are called leafs. The fan is balanced if it partitions $\mathbb{R}^{d}$ into three convex components and no two halfplanes are coplanar. In the following all our fans will be balanced. Given fans $\mathcal{F}^{0}, \ldots, \mathcal{F}^{s}$ with leafs $L_{j}^{r}$, their intersection is the collection of cones

$$
L_{i_{0}}^{0} \cap \cdots \cap L_{i_{s}}^{s}
$$

for $i_{0}, \ldots, i_{s}=1,2,3$. Define $U(d, k)$ to be the maximum of the number of inclusion-maximal cones for the intersection of $k$ fans in general position in $\mathbb{R}^{d}$. Burton conjectured the following.

Conjecture 2 ([6, Conj. 5.5.14]). For $d \geq 2, U(d, d-1)=1+2^{d-1}$.

To see why Theorem 1.4 settles Conjecture 2 , let $P=\operatorname{conv}\left(u_{1}, u_{2}, u_{3}\right) \subset \mathbb{R}^{d}$ be a triangle. The normal fan of $P$ is the collection of cones $N_{i}=\left\{\ell \in\left(\mathbb{R}^{d}\right)^{*}: \ell\left(u_{i}\right) \geq \ell\left(u_{j}\right)\right.$ for all $\left.j\right\}$ for $i=1,2,3$. These are polyhedral cones given as the intersection of exactly two distinct linear halfspaces and and hence the intersections $N_{i} \cap N_{j}$ for $i \neq j$ are halfplanes. Thus, every triangle furnishes a fan in the sense of Burton and it is easy to see that every balanced fan arises this way. Thus every collection of fans in relative general position corresponds to a collection of triangles $P_{0}, P_{1}, \ldots, P_{s} \subset \mathbb{R}^{d}$ in relative general position. The totally mixed faces of the Minkowski sum $P_{0}+\cdots+P_{s}$ are exactly the faces that arise as the Minkowski sum of an edge from each $P_{r}$. Every linear function maximizing on such a totally mixed face is thus in the intersection of one leave from each $P_{i}$.

Corollary 1.5. The number of inclusion-maximal cones in the intersection of $d-1$ fans in $\mathbb{R}^{d}$ is at most $1+2^{d-1}$.

1.4. Minkowski transforms. We close in Section 4 with an alternative construction of colorful configurations associated to Minkowski sums via projections of polytopes. These are constructed using McMullen's transforms [19]. In contrast to the colorful Gale transforms, these Minkowski transforms are realized in lower dimensional spaces but at the expense of not containing information about Minkowski subsums. In particular, their construction uses inequality descriptions of polytopes and not vertex sets, as in the case of colorful Gale transforms.

Acknowledgements. The topological part of this paper started in Berlin in 2014 and was independently completed in Berlin/Paris and Jerusalem. This paper is an amalgamation of our efforts. K. Adiprasito was supported by NSF Grant DMS 1128155 and ERCAG 320924. P. Brinkmann was supported by DFG within the research training group "Methods for Discrete Structures" (GRK1408). A. Padrol thanks the support of the INSMI (CNRS) through the program PEPS Jeunes Chercheur-e-s 2016. P. Paták's research was supported by the ERC Advanced grant no. 320924. Z. Patáková's work was supported by the Israel Science Foundation grant ISF-768/12. R. Sanyal was supported by the DFG Collaborative Research Center SFB/TR 109 "Discretization in Geometry and Dynamics".

\section{An UPPER BOUND FOR COLORFUl Simplicial DEPTH}

In this section we will cast the problem of finding tight upper bounds on the colorful simplicial depth into one of combinatorial topology. More precisely, we will relate the bound of Theorem 1.2 to the Betti numbers of certain simplicial complexes (Proposition 2.2). To set the stage, fix some $\mathbf{n}=\left(n_{0}, \ldots, n_{d}\right) \in \mathbb{Z}^{d+1}$ with $n_{i} \geq 2$ and define the $\mathbf{n}$-colorful complex as the simplicial complex

$$
\mathcal{K}=\mathcal{K}(\mathbf{n}):=\mathcal{V}_{0} * \cdots * \mathcal{V}_{d}
$$


where $\mathcal{V}_{i}$ is a 0 -dimensional complex on $n_{i}$ vertices and $*$ denotes the join. We identify $\mathcal{V}_{i}$ with its set of vertices and we assume that $\mathcal{V}_{i} \cap \mathcal{V}_{j}=\varnothing$. This way, the vertex set of $\mathcal{K}$ is $\mathcal{V}:=\mathcal{V}_{0} \cup \cdots \cup \mathcal{V}_{d}$. The simplices $\sigma \in \mathcal{K}$, called colorful simplices, are exactly the subsets $\sigma \subset \mathcal{V}$ with $\left|\sigma \cap \mathcal{V}_{i}\right| \leq 1$ for all $0 \leq i \leq d$.

In this language, a colorful n-configuration corresponds to a map $C: \mathcal{V} \rightarrow \mathbb{R}^{d}$, which induces a map $C: \mathcal{K} \rightarrow \mathbb{R}^{d}$ that linearly interpolates on each simplex $\sigma \in \mathcal{K}$ and thus

$$
C(\sigma):=\operatorname{conv}(C(v): v \in \sigma) .
$$

Conversely, any map $C: \mathcal{K} \rightarrow \mathbb{R}^{d}$ that is linear on each simplex gives a colorful configuration by setting $C_{i}:=\left\{C(v): v \in \mathcal{V}_{i}\right\}$ and we will identify colorful configurations and affine maps from $\mathcal{K}$ to $\mathbb{R}^{d}$. Thus $C$ is a centered colorful configuration if $0 \in \operatorname{relint} \operatorname{conv} C\left(\mathcal{V}_{i}\right)$ for $0 \leq i \leq d$, and $C$ is in relative general position if $0 \notin C(\tau)$ for any simplex $\tau \in \mathcal{K}$ of dimension $<d$.

The avoiding complex associated to $C$ is the simplicial subcomplex

$$
\mathcal{A}=\mathcal{A}(C):=\{\sigma \in \mathcal{K}: 0 \notin C(\sigma)\} .
$$

We define the hitting set $\mathcal{H}=\mathcal{H}(C)$ of $C$ to be the set of inclusion-minimal faces of $\mathcal{K} \backslash \mathcal{A}$. If $C$ is in general position, then $\mathcal{H}=\mathcal{K} \backslash \mathcal{A}$ and $\mathcal{H}=\left\{\eta_{1}, \ldots, \eta_{m}\right\}$ is a collection of $d$-dimensional simplices of $\mathcal{K}$, the hitting $d$-simplices of $C$. In particular, the colorful simplicial depth of a configuration $C$ in relative general position is $\operatorname{depth}(C)=|\mathcal{H}(C)|$.

For a simplicial complex $\mathcal{S}$, we write $\mathrm{C}_{*}(\mathcal{S})$ for the chain complex of $\mathcal{S}$ with coefficients in $\mathbb{Z}_{2}$. That is, for $-1 \leq k \leq \operatorname{dim} \mathcal{S}, \mathrm{C}_{k}(\mathcal{S})$ is the $\mathbb{Z}_{2}$-vector space with basis given by the $k$-dimensional faces $\sigma$ of $\mathcal{S}$. We will freely abuse notation and treat $k$-chains of $\mathcal{S}$ as collections of $k$-dimensional faces. The boundary map is given by

$$
\partial \sigma=\sum \tau
$$

where the sum is over all faces $\tau \subset \sigma$ with $\operatorname{dim} \tau=\operatorname{dim} \sigma-1$. The $k$-th homology group is denoted by $\tilde{\mathrm{H}}_{k}(\mathcal{S})=\operatorname{ker} \partial_{k} / \operatorname{im} \partial_{k+1}$ and we write $\tilde{\beta}_{k}(\mathcal{S})=\operatorname{dim}_{\mathbb{Z}_{2}} \tilde{\mathrm{H}}_{k}(\mathcal{S})$ for the $k$-th reduced Betti number of $\mathcal{S}$. We will write $\mathbf{S}^{d}$ for the sphere of dimension $d$.

Proposition 2.1. Let $\mathbf{n}=\left(n_{0}, \ldots, n_{d}\right)$ with $n_{i} \geq 2$ for all $0 \leq i \leq d$. Then

$$
\tilde{\beta}_{k}(\mathcal{K}(\mathbf{n}))=0 \quad \text { for } k<d \quad \text { and } \quad \tilde{\beta}_{d}(\mathcal{K}(\mathbf{n}))=\left(n_{0}-1\right)\left(n_{1}-1\right) \cdots\left(n_{d}-1\right) .
$$

Proof. For $0 \leq i \leq d$, the complex $\mathcal{V}_{i}$ is a wedge of $n_{i}-1$ spheres of dimension 0 . The complex $\mathcal{K}$ is by construction a join of $\mathcal{V}_{0}, \ldots, \mathcal{V}_{d}$ and, since the join distributes over wedge (see [17, Ex. 6.2.1]), we have

$$
\mathcal{K} \simeq \bigvee_{\left(n_{0}-1\right) \cdots\left(n_{d}-1\right)} \mathbf{S}^{d}
$$

This proves the claim.

The main connection between the colorful simplicial depth of $C$ and the topology of $\mathcal{A}(C)$ is the following lemma.

Lemma 2.2. For $\mathbf{n}=\left(n_{0}, \ldots, n_{d}\right)$ with $n_{i} \geq 2$, let $C$ be a centered colorful $\mathbf{n}$-configuration in relative general position. Then

$$
\operatorname{depth}(C) \leq \prod_{i=0}^{d}\left(n_{i}-1\right)+\tilde{\beta}_{d-1}(\mathcal{A}) .
$$


Proof. Let $\mathcal{A}=\mathcal{A}(C)$ be the avoiding complex of $C$. Using the Euler-Poincaré formula, we compute the reduced Euler characteristic $\tilde{\chi}(\mathcal{A})$ in two different ways. The avoiding complex $\mathcal{A}$ and the colorful complex $\mathcal{K}=\mathcal{K}(\mathbf{n})$ agree on all faces up to dimension $d-1$ and $\mathcal{A}$ is only missing the hitting simplices $\mathcal{H}=\mathcal{H}(C)$. Thus, by Proposition 2.1,

$$
\tilde{\chi}(\mathcal{A})=\tilde{\chi}(\mathcal{K})+(-1)^{d-1}|\mathcal{H}|=(-1)^{d} \prod_{i=0}^{d}\left(n_{i}-1\right)+(-1)^{d-1} \operatorname{depth}(C) .
$$

On the other hand, $\tilde{\mathrm{H}}_{k}(\mathcal{A})=\tilde{\mathrm{H}}_{k}(\mathcal{K})$ for $k<d-1$ and from Proposition 2.1 we get

$$
\tilde{\chi}(\mathcal{A})=(-1)^{d-1} \tilde{\beta}_{d-1}(\mathcal{A})+(-1)^{d} \tilde{\beta}_{d}(\mathcal{A}) .
$$

This yields

$$
\operatorname{depth}(C)=\prod_{i=0}^{d}\left(n_{i}-1\right)+\tilde{\beta}_{d-1}(\mathcal{A})-\tilde{\beta}_{d}(\mathcal{A})
$$

By way of Lemma 2.2, Theorem 1.2 follows from the main result of this section.

Theorem 2.3. Let $\mathbf{n}=\left(n_{0}, \ldots, n_{d}\right)$ with $n_{i} \geq 2$. Then $\tilde{\beta}_{d-1}(\mathcal{A}(C))=1$ for any centered colorful $\mathbf{n}$-configuration $C$ in relative general position.

We note that $\tilde{\beta}_{d-1}(\mathcal{A}(C))$ is never trivial for centered configurations in relative general position because $\mathcal{A}(C)$ retracts onto the boundary of any hitting simplex.

Proposition 2.4. Let $C$ be a centered colorful configuration in relative general position. Then

$$
\tilde{\beta}_{d-1}(\mathcal{A}(C)) \geq 1 \text {. }
$$

Proof. By construction, the avoiding complex $\mathcal{A}=\mathcal{A}(C)$ satisfies $C(\mathcal{A}) \subset \mathbb{R}^{d} \backslash\{0\}$. Hence, if $C$ is followed by the radial projection of $\mathbb{R}^{d} \backslash\{0\}$ onto the $(d-1)$-sphere $\mathbf{S}^{d-1}$, we obtain a continuous map $\hat{C}: \mathcal{A} \rightarrow \mathbf{S}^{d-1}$. By the colorful Carathéodory Theorem 1.1, there is at least one colorful $d$-simplex $\eta \in \mathcal{H}=\mathcal{K} \backslash \mathcal{A}$. The assumption of relative general position yields $0 \in \operatorname{int} C(\eta)$. Let $\Sigma$ be the boundary complex of $\eta$. Since the skeleta of $\mathcal{K}$ and $\mathcal{A}$ coincide in all dimensions $\leq d-1$, we have that $\Sigma \subseteq \mathcal{A}$ is a subcomplex and $\hat{C}$ restricted to $\Sigma$ is a homeomorphism. Thus,

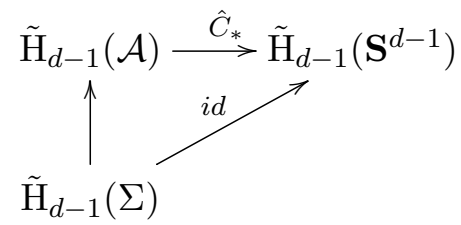

commutes and, in particular, $\hat{C}$ is surjective in homology. This shows that $\tilde{\beta}_{d-1}(\mathcal{A})>0$.

The proof of Proposition 2.4 actually shows that the boundary of any hitting simplex yields a non-trivial class in $\tilde{\mathrm{H}}_{d-1}(\mathcal{A}(C))$. The following proposition shows that the hitting simplices even generate.

Proposition 2.5. Let $C$ be a centered colorful configuration in relative general position with avoiding complex $\mathcal{A}=\mathcal{A}(C)$ and hitting set $\mathcal{H}=\mathcal{H}(C)$. Then $\tilde{\mathrm{H}}_{d-1}(\mathcal{A})$ is generated by the cycles $\partial \eta$ for $\eta \in \mathcal{H}$. 
Proof. Since $\tilde{\mathrm{H}}_{d-1}(\mathcal{K})=0$, we have, by definition, im $\partial_{d}=\operatorname{ker} \partial_{d-1}$. Since $\mathrm{C}_{k}(\mathcal{K})=\mathrm{C}_{k}(\mathcal{A})$ for $k \leq d-1$ and

the result follows.

$$
\mathrm{C}_{d}(\mathcal{K})=\mathrm{C}_{d}(\mathcal{A}) \oplus \mathbb{Z}_{2} \mathcal{H}
$$

As a consequence of Proposition 2.5 and Proposition 2.4, we note the following useful reformulation of Theorem 2.3.

Corollary 2.6. Let $C$ be a centered colorful configuration in relative general position with avoiding complex $\mathcal{A}=\mathcal{A}(C)$. Then $\tilde{\beta}_{d-1}(\mathcal{A})=1$ if and only if any two hitting simplices $\eta_{1}, \eta_{2} \in \mathcal{H}(C)$ are homologous. That is, there is some $B \in \mathrm{C}_{d}(\mathcal{A})$ such that

$$
\partial \eta_{1}+\partial \eta_{2}=\partial B
$$

Let $C, C^{\prime}: \mathcal{K} \rightarrow \mathbb{R}^{d}$ be two centered colorful configurations in relative general position. We say that $C^{\prime}$ is obtained from $C$ by a flip if there is a continuous family $\left(C_{t}: \mathcal{K} \rightarrow \mathbb{R}^{d}\right)_{-1 \leq t \leq 1}$ of centered configurations such that $C_{-1}=C$ and $C_{1}=C^{\prime}$ and $C_{t}$ is in relative general position at all times except for $t=0$. At time $t=0$, the unique minimal face $\rho \in \mathcal{K}$ with $0 \in \operatorname{relint} C_{0}(\rho)$ is of dimension $d-1$. To emphasize $\rho$, we shall say that $C$ and $C^{\prime}$ are related by a flip over $\rho$ and we write $C \stackrel{\rho}{\rightsquigarrow} C^{\prime}$. Informally, think of a flip as a continuous motion of $C$ to $C^{\prime}$ such that the origin passes through the ridge $\rho$.

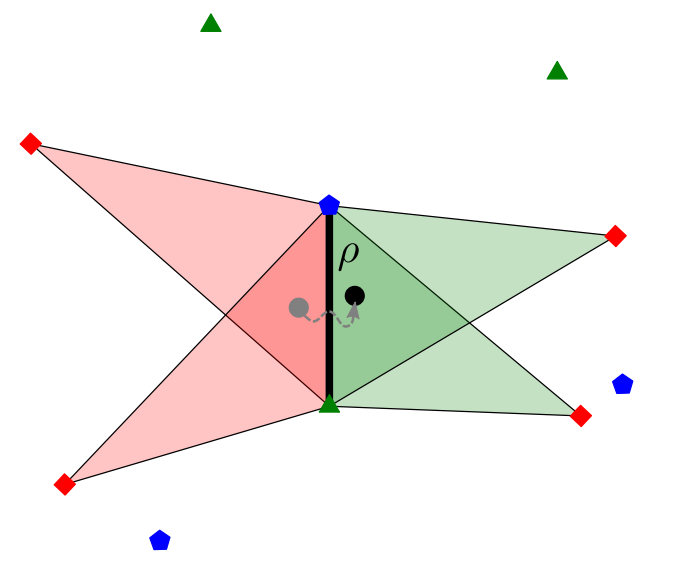

Figure 2. One can sometimes think of a $\rho$-flip as if the origin moved continuously through $\rho$ without crossing any other colorful ridge. Colorful simplices containing $\rho$ change their status, the remaining are not affected.

Proposition 2.7. Let $C \stackrel{\rho}{\rightsquigarrow} C^{\prime}$ be two configurations in relative general position related by a flip in $\rho$ and let $\mathcal{A}, \mathcal{A}^{\prime}$ and $\mathcal{H}, \mathcal{H}^{\prime}$ be the respective avoiding complexes and hitting sets. A colorful d-simplex $\sigma$ is in the symmetric difference $\mathcal{H} \triangle \mathcal{H}^{\prime}=\left(\mathcal{H} \backslash \mathcal{H}^{\prime}\right) \cup\left(\mathcal{H}^{\prime} \backslash \mathcal{H}\right)$ if and only if $\rho \subset \sigma$. Thus, a colorful d-simplex $\sigma \in \mathcal{A} \cup \mathcal{A}^{\prime}$ is contained in $\mathcal{A} \cap \mathcal{A}^{\prime}$ if and only if $\rho$ is not a face of $\sigma$.

Proof. Let $\left(C_{t}\right)_{-1 \leq t \leq 1}$ be the continuous family that realizes the flip over $\rho$. For $\varepsilon>0$ sufficiently small, every colorful $d$-simplex $\sigma$ that contains $\rho$ is hitting for $C_{-\varepsilon}$ if and only if it is not hitting for $C_{+\varepsilon}$. Hence, the colorful simplices in the symmetric difference $\mathcal{H} \triangle \mathcal{H}^{\prime}$ are exactly the colorful $d$-simplices that contain $\rho$. 
The benefit of working with flips is that we can pass from one colorful configuration to another.

Proposition 2.8. Any two centered colorful $\mathbf{n}$-configurations in relative general position are connected by a sequence of flips.

Proof. Let us denote by $\mathbf{F} \subset\left\{C: \mathcal{V} \rightarrow \mathbb{R}^{d}\right\}$ the space of all centered colorful configurations. This is an open semialgebraic set of full dimension $d\left(n_{0}+\cdots+n_{d}\right)$. The set $X \subset \mathbb{R}^{\mathcal{V}}$ of colorful configurations $C$ such that $0 \in C\left(\rho_{1}\right) \cap C\left(\rho_{2}\right)$ for two distinct colorful $(d-1)$-simplices $\rho_{1}, \rho_{2} \in \mathcal{K}$ is of codimension 2 . Indeed, if we forget about containment and only require that $0 \in$ aff $C\left(\rho_{1}\right) \cap$ aff $C\left(\rho_{2}\right)$, then the dimension can only increase. For two fixed $\rho_{1}$ and $\rho_{2}$, pick $a_{1} \in \rho_{1} \backslash \rho_{2}$ and $a_{2} \in \rho_{2} \backslash \rho_{1}$. We can choose $C(p) \in \mathbb{R}^{d}$ freely for any $p \in \mathcal{V} \backslash\left\{a_{1}, a_{2}\right\}$ and we have to choose $C\left(a_{i}\right)$ in the linear span of $\left\{f(p): p \in \rho_{i}, p \neq a_{i}\right\}$, which is a linear space of dimension at most of dimension $d-2$. This yields $d\left(n_{0}+\cdots+n_{d}\right)-2$ degrees of freedom and $X$ is contained in a finite union of these sets as $\rho_{1}$ and $\rho_{2}$ vary over all distinct $(d-1)$-simplices of $\mathcal{K}$. It follows that $\mathbf{F} \backslash X$ is path connected, which proves the claim.

The crucial insight now is that $\tilde{\beta}_{d-1}(\mathcal{A}(C))$ is invariant as $C$ undergoes a flip.

Lemma 2.9. Let $C \stackrel{\rho}{\rightsquigarrow} C^{\prime}$ be two centered colorful configurations in general position that are related by a flip in $\rho$. If $\beta_{d-1}(\mathcal{A}(C))=1$, then $\beta_{d-1}\left(\mathcal{A}\left(C^{\prime}\right)\right)=1$.

Proof. Let $\mathcal{A}, \mathcal{A}^{\prime}$ be the avoiding complexes of $C$ and $C^{\prime}$, respectively, and write $\mathcal{H}$ and $\mathcal{H}^{\prime}$ for their hitting sets. In light of Corollary 2.6, we want to show that for any $\eta_{1}, \eta_{2} \in \mathcal{H}^{\prime}$ there is a $d$-chain $B^{\prime} \in \mathrm{C}_{d}\left(\mathcal{A}^{\prime}\right)$ such that $\partial \eta_{1}+\partial \eta_{2}=\partial B^{\prime}$. For the proof, let us call a hitting simplex $\eta$ old if $\eta \in \mathcal{H} \cap \mathcal{H}^{\prime}$ and new if $\eta \in \mathcal{H}^{\prime} \backslash \mathcal{H}$. By Proposition 2.7, a hitting simplex $\eta$ is new if and only if $\rho \subseteq \eta$. Without loss of generality, $\rho=\left\{a_{1}, a_{2}, \ldots, a_{d}\right\}$ with $a_{i} \in \mathcal{V}_{i}$ for $1 \leq i \leq d$. That is, $\rho$ is a colorful ridge that avoids $\mathcal{V}_{0}$. Our proof distinguishes three cases, depending on whether $\eta_{1}$ and $\eta_{2}$ are new or old. It follows from the (polyhedral) Morse Lemma that only new hitting simplices can create homology and, strictly speaking, we only need to consider the 'new/new' case. However, to have a self-contained proof, we include all cases.

New/New: Assume that $\eta_{1}, \eta_{2} \in \mathcal{H}^{\prime} \backslash \mathcal{H}$. Then there are $a_{0}, b_{0} \in \mathcal{V}_{0}$ such that $\eta_{1}=a_{0} * \rho=$ $\left\{a_{0}, a_{1}, \ldots, a_{d}\right\}$ and $\eta_{2}=b_{0} * \rho$. Let us consider the critical configuration $C_{t}$ at time $t=0$. Let $H$ be the linear hyperplane spanned by $C_{0}(\rho)$ and denote by $H^{+}$the closed halfspace containing $C_{0}\left(a_{0}\right)$ and $C_{0}\left(b_{0}\right)$. The points must lie in the same halfspace, because $\eta_{1}$ and $\eta_{2}$ are hitting in $C_{\varepsilon}$ for $\varepsilon>0$. Since $C$ is centered, there are $b_{i} \in \mathcal{V}_{i}$ such that $C_{0}\left(b_{i}\right) \in H^{+}$for all $1 \leq i \leq d$. Define the subcomplex

$$
\mathrm{O}\left(\eta_{1}, \eta_{2}\right):=\left\{a_{0}, b_{0}\right\} *\left\{a_{1}, b_{1}\right\} * \cdots *\left\{a_{d}, b_{d}\right\} \subset \mathcal{K},
$$

which, as an abstract simplicial complex, is the boundary of a $(d+1)$-dimensional crosspolytope. Except for $\eta_{1}$ and $\eta_{2}$, every simplex $\sigma \in \mathrm{O}\left(\eta_{1}, \eta_{2}\right)$ belongs to $\mathcal{A} \cap \mathcal{A}^{\prime}$. Indeed, they are avoiding already in $C_{0}$ by construction, because they lie all in the same linear halfspace and only $\rho$ contains 0 in its relative interior. Moreover, every avoiding simplex of $C_{0}$ is also an avoiding simplex of $C_{t}$ for $-1 \leq t \leq 1$, since only the simplices that contain $\rho$ as a face change their status in the course of the flip. We call the $d$-chain

$$
\mathrm{Z}\left(\eta_{1}, \eta_{2}\right):=\sum\left\{\sigma: \sigma \in \mathrm{O}\left(\eta_{1}, \eta_{2}\right) \backslash\left\{\eta_{1}, \eta_{2}\right\}, \operatorname{dim}(\sigma)=d\right\} \in \mathrm{C}_{d}\left(\mathcal{A}^{\prime}\right)
$$

a cylinder of $\eta_{1}$ and $\eta_{2}$ and we observe that

$$
0=\partial \mathrm{O}\left(\eta_{1}, \eta_{2}\right)=\partial \mathrm{Z}\left(\eta_{1}, \eta_{2}\right)+\partial \eta_{1}+\partial \eta_{2},
$$


which shows that $\partial \eta_{1}+\partial \eta_{2}$ is a boundary and finishes the first case.

Old/Old: Assume that $\eta_{1}, \eta_{2} \in \mathcal{H}^{\prime} \cap \mathcal{H}$ and thus $\rho$ is not a face of neither $\eta_{1}$ nor $\eta_{2}$. Since $\eta_{1}$ and $\eta_{2}$ are hitting for $C$, the assumption and Corollary 2.6 assure us that there is a $d$-chain $B \in \mathrm{C}_{d}(\mathcal{A})$ such that

$$
\partial \eta_{1}+\partial \eta_{2}=\partial B=\partial\left(\sum_{\sigma \in B \cap \mathcal{A}^{\prime}} \sigma+\sum_{\sigma \in B \cap \mathcal{H}^{\prime}} \sigma\right) .
$$

We claim that the number of simplices in $B \cap \mathcal{H}^{\prime}$ is even. To this end, consider the cochain $\rho^{*}: \mathrm{C}_{d-1}(\mathcal{K}) \rightarrow \mathbb{Z}_{2}$ with $\rho^{*}(\rho)=1$ and $=0$ otherwise. The coboundary $\delta \rho^{*}=\rho^{*} \circ \partial$ satisfies that for a colorful $d$-simplex $\sigma \in \mathcal{K}$

$$
\delta \rho^{*}(\sigma)=1 \quad \text { if } \rho \subset \sigma \quad \text { and } \quad \delta \rho^{*}(\sigma)=0 \quad \text { otherwise. }
$$

Since neither $\eta_{1}$ nor $\eta_{2}$ contain $\rho$, we compute from (2)

$$
0=\rho^{*}\left(\partial \eta_{1}+\partial \eta_{2}\right)=\delta \rho^{*}(B)=\sum_{\sigma \in B \cap \mathcal{A}^{\prime}} \delta \rho^{*}(\sigma)+\sum_{\sigma \in B \cap \mathcal{H}^{\prime}} \delta \rho^{*}(\sigma) .
$$

Because $\delta \rho^{*}$ evaluates to 0 on the simplices of $B \cap \mathcal{A}^{\prime} \subseteq \mathcal{A} \cap \mathcal{A}^{\prime}$ and to 1 on the simplices of $B \cap \mathcal{H}^{\prime} \subset \mathcal{H}^{\prime} \backslash \mathcal{H}$ (cf. Proposition 2.7), this shows that $\left|B \cap \mathcal{H}^{\prime}\right|$ is even. Let $B \cap \mathcal{H}^{\prime}=$ $\left\{\sigma_{1}, \ldots, \sigma_{2 k}\right\}$. Since each $\sigma_{i}$ is a new hitting simplex, we can use the previous case to find cylinders $\mathrm{Z}\left(\sigma_{2 i-1}, \sigma_{2 i}\right) \in \mathrm{C}_{d}\left(\mathcal{A}^{\prime}\right)$ such that

$$
\partial \sum_{\sigma \in B \cap \mathcal{H}^{\prime}} \sigma=\sum_{i=1}^{k} \partial\left(\sigma_{2 i-1}+\sigma_{2 i}\right)=\sum_{i=1}^{k} \partial \mathrm{Z}\left(\sigma_{2 i-1}, \sigma_{2 i}\right),
$$

which together with (2) shows that $\partial \eta_{1}+\partial \eta_{2}$ is a boundary in $\mathcal{A}^{\prime}$.

Old/New: Assume that $\eta_{1} \in \mathcal{H}^{\prime} \cap \mathcal{H}$ and $\eta_{2} \in \mathcal{H}^{\prime} \backslash \mathcal{H}$ and thus $\rho \nsubseteq \eta_{1}$ but $\rho \subseteq \eta_{2}$. Again, let $a \in \mathcal{V}_{0}$ such that $\eta_{2}=a * \rho$. Let $H$ be the linear hyperplane parallel to aff $\left(C^{\prime}(\rho)\right)$. Since $C^{\prime}$ is in relative general position, $C^{\prime}\left(\eta_{2}\right)$ is a $d$-simplex and $H$ separates $C^{\prime}(a)$ from $C^{\prime}(\rho)$. By the centeredness, there must be some $b \in \mathcal{V}_{0}$ such that $H$ separates $C^{\prime}(a)$ and $C^{\prime}(b)$. In particular, the colorful simplex $\eta_{2}^{\prime}:=b * \rho$ is contained in $\mathcal{H} \backslash \mathcal{H}^{\prime}$; that is, $\eta_{2}^{\prime}$ an element of $\mathcal{A}^{\prime}$ containing $\rho$.

Hence, $\eta_{1}, \eta_{2}^{\prime}$ is a pair of hitting simplices for $C$ and, by the assumption and Corollary 2.6, there is a chain $B \in \mathrm{C}_{d}(\mathcal{A})$ such that $\partial \eta_{1}+\partial \eta_{2}^{\prime}=\partial B$. Define $B^{\prime}:=B+\eta_{2}+\eta_{2}^{\prime}$. This is a chain in $\mathrm{C}_{d}(\mathcal{K})$ and

$$
\partial B^{\prime}=\partial B+\partial \eta_{2}+\partial \eta_{2}^{\prime}=\partial \eta_{1}+\partial \eta_{2}
$$

Using the cochain $\rho^{*}$, we observe that $\rho^{*}\left(\partial \eta_{1}+\partial \eta_{2}^{\prime}\right)=1$ and hence $B \cap \mathcal{H}^{\prime}$ contains an odd number of elements. Since $\eta_{2} \in \mathcal{H}^{\prime}$ and $\eta_{2}^{\prime} \notin \mathcal{H}^{\prime}$, it follows that $B^{\prime}=B+\eta_{2}+\eta_{2}^{\prime}$ contains an even number of elements in $\mathcal{H}^{\prime}$ and, by pairing them up with cylinders, there is some $B^{\prime \prime} \in \mathrm{C}_{d}\left(\mathcal{A}^{\prime}\right)$ such that $\partial B^{\prime}=\partial B^{\prime \prime}$.

Proof of Theorem 2.3. By Lemma 2.9 and Proposition 2.8, it suffices to produce a single instance of a centered colorful configuration in relative general position for every $\mathbf{n}$ whose avoiding complex satisfies $\tilde{\beta}_{d-1}(\mathcal{A})=1$.

Note that the centered configurations of Example 1.3 are in relative general position. From the description of the hitting simplices in Example 1.3, we can get their avoiding complexes. For $0 \leq i \leq d$, let $\mathcal{V}_{i}$ be the 0 -dimensional complex with vertices $a^{i}, b_{1}^{i}, \ldots, b_{n_{i}-1}^{i}$. Then $\sigma \in \mathcal{K}(\mathbf{n})=\mathcal{V}_{0} * \cdots * \mathcal{V}_{n}$ is an avoiding $d$-simplex if and only if it contains at least one $a^{i}$ and at least one $b_{l}^{j}$ for $i \neq j$ and $1 \leq l<n_{j}$. We call the vertices $a^{i}$ special and we set $\mathcal{A}_{d+1}$ to be 
the boundary complex of the hitting simplex $\left\{a^{0}, \ldots, a^{d}\right\}$. We will show that $\mathcal{A}$ is homotopy equivalent to $\mathcal{A}_{d+1}$ and hence $\tilde{\mathrm{H}}_{*}(\mathcal{A})=\tilde{\mathrm{H}}_{*}\left(\mathbf{S}^{d-1}\right)$.

Recall that if $\sigma$ is an inclusion-maximal face of a simplicial complex $\Delta$, and $\tau \subset \sigma$ is a face with $\operatorname{dim} \tau<\operatorname{dim} \sigma$ that is not contained in any other inclusion-maximal face of $\Delta$, then $\tau$ is called a free face of $\sigma$. If $\tau$ is a free face of $\sigma$, the removal of all the simplices $\tau \subseteq \gamma \subseteq \sigma$ of $\Delta$ is called a collapse, and denoted by $\Delta \searrow \Delta^{\prime}$, where $\Delta^{\prime}=\Delta \backslash\{\gamma: \tau \subseteq \gamma \subseteq \sigma\}$. We will use the same notation to denote a sequence of collapses. If $\Delta \searrow \Delta^{\prime}$ then $\Delta$ and $\Delta^{\prime}$ are homotopy equivalent; cf. [5, Sect. 11.1].

For $1 \leq k \leq d$, let $\mathcal{A}_{k} \subseteq \mathcal{A}$ be the subcomplex generated by the $d$-simplices with at least $k$ special vertices. We will show that

$$
\mathcal{A}=\mathcal{A}_{1} \searrow \mathcal{A}_{2} \searrow \cdots \searrow \mathcal{A}_{d} \searrow \mathcal{A}_{d+1} \cong \mathbf{S}^{d-1}
$$

For $1 \leq k \leq d-1$, let $\sigma \in \mathcal{A}_{k}$ be a $d$-simplex containing exactly $k$ special vertices and let $\tau \subset \sigma$ be any ridge containing $k-1$ special vertices. Since every $\mathcal{V}_{i}$ contains exactly one special vertex and $\tau$ misses only one color class $\mathcal{V}_{i}, \tau$ is a free face of $\sigma$. Hence, it can be collapsed. Continuing this way shows $\mathcal{A}_{k} \searrow \mathcal{A}_{k+1}$. In the last step, the simplices $\sigma \in \mathcal{A}_{d}$ contain a single non-special vertex, which is itself a free face of $\sigma$ and can be collapsed. What remains are the $(d-1)$-simplices spanned by special vertices, which is exactly $\mathcal{A}_{d+1}$.

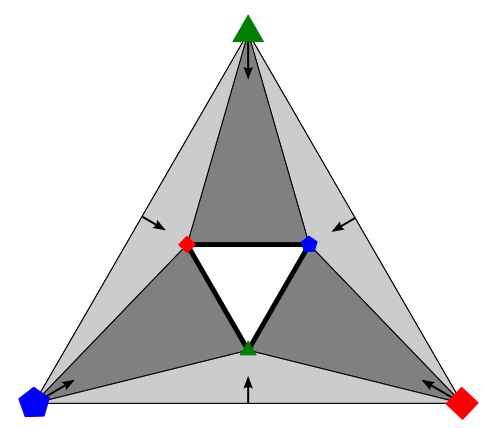

Figure 3 . Scheme of $\mathcal{A}$ for Example 1.3 for $d=2$. The large nodes on the outer triangle should be thought of as representatives of the non-special vertices of that color. Since no simplex of $\mathcal{A}$ has two vertices of the same color, this depicts the whole complex. Lightly shaded triangles belong to $\mathcal{A}_{1}$ and darkly shaded triangles to $\mathcal{A}_{2}$. The boundary of the interior triangle, drawn thicker, is $\mathcal{A}_{3}$. The collapses for the proof of Theorem 2.3 are also depicted.

The Universal Coefficient Theorem together with Hurewicz' theorem (cf. [12]) shows that all homotopy groups of $\mathcal{A}(C)$ up to dimension $d-1$ agree with that of the $(d-1)$-sphere.

Conjecture. For a centered colorful configuration $C$ in relative general position, the avoiding complex $\mathcal{A}(C) d$-collapses (in the sense of Wegner [27]) onto the boundary of a hitting simplex.

For the Colorful Carathéodory Theorem, the assumption of centeredness can be replaced by a weaker assumption (see, for example, $[2,3,13,20]$ ). This prompts the following question.

Question 1. Under which conditions other than centeredness is $\tilde{\beta}_{d-1}(\mathcal{A})=1$ ? 


\section{From COlorful CONFigurations to Minkowski sums}

In this section we will show how to use Gale duality and the Cayley trick to translate between colorful configurations and Minkowski sums. Although we are mainly interested in the case of polytopes, our setup works with arbitrary point configurations (not necessarily in convex position). Let $\mathcal{V}$ be a finite set. A point configuration in $\mathbb{R}^{d}$ indexed by $\mathcal{V}$ is a map $A: \mathcal{V} \rightarrow \mathbb{R}^{d}$. Note that our point configurations can have multiple points. A subset $\mathcal{U} \subseteq \mathcal{V}$ is a face of $A$ if there is an affine function $\ell: \mathbb{R}^{d} \rightarrow \mathbb{R}$ such that $\ell(A(v)) \geq 0$ and $>0$ if and only if $v \notin \mathcal{U}$. That is, we identify faces of the convex hull conv $A(\mathcal{V})$ with the indices of points contained in them.

If we further assume that the affine span of $A(\mathcal{V})$ is full-dimensional, then $A$ induces a surjective linear map $\widehat{A}: \mathbb{R}^{\mathcal{V}} \rightarrow \mathbb{R}^{d+1}$ by sending $e_{v} \mapsto(A(v), 1)$ for $v \in \mathcal{V}$ and extending by linearity. The map $\widehat{A}$ has a (possibly trivial) $\operatorname{kernel} \operatorname{ker}(\hat{A}) \cong \mathbb{R}^{|\mathcal{V}|-d-1}$ and let $\iota: \operatorname{ker}(\widehat{A}) \hookrightarrow \mathbb{R}^{\mathcal{V}}$ denote the inclusion map. The adjoint to $\iota$ is a linear map

$$
\iota^{*}:\left(\mathbb{R}^{\mathcal{V}}\right)^{*} \rightarrow(\operatorname{ker}(\widehat{A}))^{*} .
$$

Identifying $(\operatorname{ker}(\widehat{A}))^{*}$ with $\mathbb{R}^{|\mathcal{V}|-d-1}$, we get another configuration $G: \mathcal{V} \rightarrow \mathbb{R}^{|\mathcal{V}|-d-1}$, the Gale transform $^{1}$ of $A$ given by $G(v)=\iota^{*}\left(e_{v}^{*}\right)$ for $v \in I$.

Lemma 3.1 (Gale duality). Let $A: \mathcal{V} \rightarrow \mathbb{R}^{d}$ be a (full-dimensional) point configuration and $G: \mathcal{V} \rightarrow \mathbb{R}^{|\mathcal{V}|-d-1}$ a Gale transform of $A$. Then $\mathcal{U} \subseteq \mathcal{V}$ is a face of $A$ if and only if

$$
0 \in \operatorname{relint} \operatorname{conv}(G(i): i \in \mathcal{V} \backslash \mathcal{U}) \text {. }
$$

Gale transforms and Gale duality are powerful tools in discrete geometry and we refer to [16, Section 5.6] for a very accessible treatment in terms of matrices and to [28, Lecture 5] for its relation to oriented matroids. In particular, the faces of the polytope conv $A(\mathcal{V})$ are completely described by the Gale transform $G$.

Let us assume that $\mathcal{V}=\mathcal{V}_{0} \cup \mathcal{V}_{1} \cup \cdots \cup \mathcal{V}_{s}$ for some $s>0$ and $\mathcal{V}_{i} \cap \mathcal{V}_{j}=\varnothing$ for $i \neq j$. Then, similar to Section 2, we consider the point configuration $A$ as a family of $s+1$ separate configurations $A_{i}: \mathcal{V}_{i} \rightarrow \mathbb{R}^{d}$. Their Minkowski sum is the point configuration:

$$
\begin{aligned}
\sum_{\mathcal{V}} A: \mathcal{V}_{0} \times \cdots \times \mathcal{V}_{s} & \rightarrow \mathbb{R}^{d} \\
\left(v_{0}, \ldots, v_{s}\right) & \mapsto A\left(v_{0}\right)+\cdots+A\left(v_{s}\right) .
\end{aligned}
$$

We can restrict this configuration to any subset $\mathcal{U} \subset \mathcal{V}$ with $\mathcal{U}_{i}=\mathcal{U} \cap \mathcal{V}_{i} \neq \varnothing$. It is easy to check that every face of $\sum A$ is of the form $\sum_{\mathcal{U}} A$ for some $\mathcal{U} \subseteq \mathcal{V}$ such that $\mathcal{U}_{i}$ is a face of $A_{i}$.

We may adapt the notion of Gale transforms to Minkowski sums by way of Cayley embeddings. The Cayley embedding of $A: \mathcal{V} \rightarrow \mathbb{R}^{d}$ is the configuration $\operatorname{Cay}_{\mathcal{V}}(A): \mathcal{V} \rightarrow \mathbb{R}^{s} \times \mathbb{R}^{d}$ that takes $v_{i} \in \mathcal{V}_{i}$ to $\left(e_{i}, A\left(v_{i}\right)\right)$ for $0 \leq i \leq s$. Here, $e_{0}:=0$ and $e_{1}, \ldots, e_{s}$ is the standard basis of $\mathbb{R}^{s}$. Alternatively, you can also replace $e_{0}, \ldots, e_{s}$ with the vertices of any full-dimensional simplex in $\mathbb{R}^{s}$.

Let $b=\frac{1}{s+1}\left(e_{0}+\cdots+e_{s}\right)$ be the barycenter of $e_{0}, \ldots, e_{s}$ and consider the affine subspace $\Lambda=\left\{(x, y) \in \mathbb{R}^{s} \times \mathbb{R}^{d}: x=b\right\}$. Then it is straightforward to check that

$$
\operatorname{conv}\left(\operatorname{Cay}_{\mathcal{V}}(A)\right) \cap \Lambda \cong \operatorname{conv}\left(\sum_{\mathcal{V}} A\right) \text {. }
$$

\footnotetext{
${ }^{1}$ Actually, $G$ is typically treated as a vector configuration.
} 
In particular, this induces a bijection between faces of $\operatorname{Cay}_{\mathcal{V}}(A)$ and $\sum_{\mathcal{V}} A$. Cayley embeddings have many favorable properties, in particular in relation to triangulations and mixed subdivisions; see [14].

Lemma 3.2. Let $\mathcal{U}=\mathcal{U}_{0} \cup \cdots \cup \mathcal{U}_{s} \subseteq \mathcal{V}$ be such that $\mathcal{U}_{i} \neq \varnothing$ for all $0 \leq i \leq s$. Then $\mathcal{U}$ is a face of $\operatorname{Cay}_{\mathcal{V}}(A)$ if and only if $\mathcal{U}_{0} \times \cdots \times \mathcal{U}_{s}$ is a face of $\sum_{\mathcal{V}} A$.

Let $G$ be a Gale transform of $\operatorname{Cay}_{\mathcal{V}}(A)$. By construction, $G$ is a colorful point configuration and we refer to it as a colorful Gale transform. If $A$ is a configuration of $N=|\mathcal{V}|=$ $\left|\mathcal{V}_{0}\right|+\cdots+\left|\mathcal{V}_{s}\right|$ points in $\mathbb{R}^{d}$, then $G$ is a colorful configuration of $N$ points in $(N-d-s-1)$ dimensional space. Since $\mathcal{V} \backslash \mathcal{V}_{j}$ is a face for all $0 \leq j \leq s$, it follows from Lemma 3.1 that $0 \in$ relint conv $G\left(\mathcal{V}_{j}\right)$ and hence $G$ is actually a centered configuration. This allows us to construct a dictionary between the facial structure of $\sum_{\mathcal{V}} A$ and properties of the colorful configuration $G$. We start with a characterization that shows that every centered colorful configuration can be thought as a colorful Gale transform.

A configuration $G: \mathcal{V} \rightarrow \mathbb{R}^{d}$ is positively spanning if $G(\mathcal{V})$ is not contained in a closed linear halfspace; and it is positively 2-spanning if for any $v \in \mathcal{V}$, the restriction of $G$ to $\mathcal{V} \backslash\{v\}$ is positively spanning. A configuration $G^{\prime}: \mathcal{V} \rightarrow \mathbb{R}^{d}$ is a positive rescaling of $G$ if there is $\lambda: \mathcal{V} \rightarrow \mathbb{R}_{>0}$ such that $G^{\prime}(v)=\lambda(v) G(v)$ for all $v \in \mathcal{V}$. We say that $G$ and $G^{\prime}$ are positively equivalent if they are related by a positive rescaling. Notice that in this case $G^{\prime}$ is centered, positively spanning or positively 2 -spanning whenever $G$ is. The Gale transform $G$ and $G^{\prime}$ of $A$ and $A^{\prime}$ are positively equivalent if and only if $A$ and $A^{\prime}$ are projectively isomorphic [11, 5.4.vi].

The following characterization of colorful Gale transforms is implicit in [21, Prop. 2.9].

Lemma 3.3. For $\mathcal{V}=\mathcal{V}_{0} \cup \cdots \cup \mathcal{V}_{s}$. The Gale transform $G: \mathcal{V} \rightarrow \mathbb{R}^{|\mathcal{V}|-d-s-1}$ of a (full-dimensional) Cayley embedding $A: \mathcal{V} \rightarrow \mathbb{R}^{s+d}$ is centered. Conversely, every (fulldimensional) centered configuration is positively equivalent to the Gale transform of a Cayley embedding.

Moreover, the points $A\left(\mathcal{V}_{j}\right)$ are in convex position for every $0 \leq j \leq s$ if and only if $G$ is positively 2-spanning.

As in the case of ordinary Gale duality, the facial structure of the Minkowski sum of a colorful configuration can be read from the colorful Gale dual. The following dictionary follows the same reasoning as for classical Gale duality and we therefore omit the proof.

Proposition 3.4 (Colorful Gale duality). Fix $\mathcal{V}=\mathcal{V}_{0} \cup \cdots \cup \mathcal{V}_{s}$. Let $A: \mathcal{V} \rightarrow \mathbb{R}^{d}$ be a configuration such that $\sum_{\mathcal{V}} A$ is full-dimensional and let $G: \mathcal{V} \rightarrow \mathbb{R}^{|\mathcal{V}|-d-s-1}$ be a corresponding colorful Gale transform. For $\mathcal{U} \subseteq \mathcal{V}$ such that $\mathcal{U}_{i} \neq \varnothing$ for all $0 \leq i \leq d$ the following holds

$$
\sum_{\mathcal{U}} A \text { is a face of } \sum_{\mathcal{V}} A \quad \text { if and only if } \quad 0 \in \operatorname{relint} G(\mathcal{V} \backslash \mathcal{U}) \text {. }
$$

Note that the colorful Gale transform actually encodes also the faces of all subsums of $A_{0}, \ldots, A_{s}$, which are in bijection to the faces of $\operatorname{Cay}_{\mathcal{V}}(A)$. Indeed, for $\mathcal{U} \subseteq \mathcal{V}$ let $I \subset\{0, \ldots, s\}$ be the set of indices such that $\mathcal{U}_{i}=\varnothing$ and set $\mathcal{V}_{I}=\bigcup_{i \in I} \mathcal{V}_{i}$ and $\mathcal{U}_{I}=\bigcup_{i \in I} \mathcal{U}_{i}$. Then $\mathcal{U}_{I}$ is a face of the Minkowski sum $\sum_{\mathcal{V}_{I}} A$ if and only if $0 \in \operatorname{relint} G(\mathcal{V} \backslash \mathcal{U})$.

In the next section we will see another way to associate a colorful configuration to a Minkowski sum. In contrast to the colorful Gale transform, that colorful configuration is realized in lower 
dimensions but it will only remember faces of the full Minkowski sums and, for example, will not contain the face structure of the summands.

The following result, which is obtained from the duality between deletions and contractions of Gale transforms [28, Section 6.3(d)], gives a glimpse at this connection by showing how to recover the Gale transform of each of the summands from the colorful Gale transform.

Proposition 3.5. For $\mathcal{V}=\mathcal{V}_{0} \cup \cdots \cup \mathcal{V}_{s}$, let $A: \mathcal{V} \rightarrow \mathbb{R}^{d}$ be a configuration and let $G$ : $\mathcal{V} \rightarrow \mathbb{R}^{|\mathcal{V}|-d-s-1}$ be an associated colorful Gale transform. For $0 \leq i \leq s$, a Gale transform of $A_{i}: \mathcal{V}_{i} \rightarrow \mathbb{R}^{d}$ is given by the projection $G_{i}: \mathcal{V}_{i} \rightarrow \mathbb{R}^{|\mathcal{V}|-d-s-1} / L$, where $L$ is the linear span of $G\left(\mathcal{V} \backslash \mathcal{V}_{i}\right)$.

In particular, the points $A_{i}$ are affinely independent if and only if $G\left(\mathcal{V} \backslash \mathcal{V}_{i}\right)$ is spanning.

Notice that this last property is always fulfilled by colorful configurations in relative general position. Indeed, if $G\left(\mathcal{V} \backslash \mathcal{V}_{i}\right)$ was not full dimensional, we could apply the Colorful Carathéodory Theorem to find a colorful ridge containing the origin.

If for all $0 \leq i \leq s$ the points of $A_{i}$ are affinely independent, then $P_{i}:=\operatorname{conv} A\left(\mathcal{V}_{i}\right)$ is a simplex and $\operatorname{conv} \sum A=P_{1}+\cdots+P_{s}$ is a Minkowski sum of simplices.

Corollary 3.6. Let $\mathcal{V}=\mathcal{V}_{0} \cup \cdots \cup \mathcal{V}_{d}$ with $n_{i}=\left|\mathcal{V}_{i}\right| \geq 2$ for all $0 \leq i \leq d$ and set $n=$ $n_{0}+\cdots+n_{d}$. Then every centered colorful configuration of $G: \mathcal{V} \rightarrow \mathbb{R}^{d}$ in relative general position is the colorful Gale transform of a configuration $A: \mathcal{V} \rightarrow \mathbb{R}^{n-2 d-1}$ such that $\sum A$ is full-dimensional and $A_{i}$ is affinely independent for all $0 \leq i \leq d$.

A converse statement also holds, for the adequate notion of general position of Minkowski sums of simplices. For example, if all the simplices are in relative general position in the sense of [1], then their colorful Gale dual is in relative general position. We do not elaborate further because we will only need Corollary 3.6 in this direction.

We are interested in the face structure of $\sum_{\mathcal{V}} A$. We will call a face $\mathcal{U}=\mathcal{U}_{0} \cup \cdots \cup \mathcal{U}_{s}$ mixed if $\operatorname{dim}\left(A\left(\mathcal{U}_{i}\right)\right) \geq 1$ for all $1 \leq i \leq s$. A facet is totally mixed if $\mathcal{U}_{i}$ is a facet of $A_{i}$ for all $0 \leq i \leq s$.

Proposition 3.4 allows to easily translate many properties between the two setups. In particular information on facets. Notice that, since facets of $A$ are inclusion-maximal faces, the corresponding sub-configuration of $G$ is an inclusion minimal subset containing the origin in its convex hull. In particular, facets of $\sum_{\mathcal{V}} A$ correspond to subsets $\mathcal{S}=\mathcal{S}_{0} \cup \cdots \cup \mathcal{S}_{s}$ with $\mathcal{S}_{i} \varsubsetneqq \mathcal{V}_{i}$ such that $G(\mathcal{S})$ is affinely independent and $0 \in \operatorname{relint}(G(\mathcal{S}))$.

Lemma 3.7. Let $\mathcal{V}=\mathcal{V}_{0} \cup \cdots \cup \mathcal{V}_{d}$ with $n_{i}=\left|\mathcal{V}_{i}\right| \geq 2$, and let $G: \mathcal{V} \rightarrow \mathbb{R}^{d}$ be a centered colorful configuration that is the colorful Gale transform of $A: \mathcal{V} \rightarrow \mathbb{R}^{n-2 d-1}$. Let $\sigma=\left\{v_{0}, \ldots, v_{s}\right\}$ be a hitting colorful simplex of $G$ such that $0 \in \operatorname{int} G(\sigma)$. Then $\bigcup \mathcal{V}_{i} \backslash\left\{v_{i}\right\}$ is a totally mixed facet of $\sum_{\mathcal{V}} A$.

If moreover each of the $A_{i}$ is affinely independent for all $0 \leq i \leq d$, the converse holds: each totally mixed facet is of $\sum_{\mathcal{V}} A$ is of the form $\bigcup \mathcal{V}_{i} \backslash\left\{v_{i}\right\}$ for a hitting colorful simplex $\sigma=\left\{v_{0}, \ldots, v_{s}\right\}$ with $0 \in \operatorname{int} G(\sigma)$.

Observe that the reciprocal does not hold in the general case, since such a totally mixed facet $\sum_{\mathcal{U}} A$ exists if and only if each $A\left(\mathcal{V}_{i}\right)$ is a pyramid with basis $A\left(\mathcal{U}_{i}\right)$. However, the case in which we are most interested in is the Minkowski sum of simplices, in which every facet is a pyramidal base. 
In particular, in light of Corollary 3.6, centered colorful $\mathbf{n}$-configurations in relative general position can be interpreted as the Cayley embedding of a collection of simplices $A_{0}, \ldots, A_{d}$ in $\mathbb{R}^{D}$, where $D=1+\sum\left(\operatorname{dim}\left(A_{i}\right)-1\right)$. The dimensions are not arbitrary: The only possibility that a generic collection of polytopes in $\mathbb{R}^{D}$ can have a totally mixed facet is that $D=$ $1+\sum\left(\operatorname{dim}\left(A_{i}\right)-1\right)$ (and then such a facet is isomorphic to a product of facets of each $A_{i}$ ).

Then Theorem 1.4 is just a result of translating Theorem 1.2 to Minkowski sums.

Proof of Theorem 1.4. Let $G$ be the colorful Gale transform of $A$. By Lemma 3.7, the number of totally mixed facets of $\sum_{\mathcal{V}} A$ is the number of hitting simplices of $G$ that contain the origin in its relative interior. Since this is an open condition, the maximum is is clearly attained by a colorful configuration in general position, and the Corollary follows directly from Theorem 1.2.

\section{Minkowski sums And Generalized Gale transforms}

In this final section we wish to outline an alternative way to associate a colorful configuration to a Minkowski sums. This is by way of generalized Gale transforms that were first described by McMullen [19]. He presented several applications in the study of zonotopes and centrally symmetric polytopes, and one purpose of this section is to show new applications of this construction. This is best done for polytopes and this section differs from the previous ones in that we focus on polytopes and their vertex sets.

Let $P \subset \mathbb{R}^{d}$ be a full-dimensional polytope. We are interested in the facial structure of $\pi(P)$, where $\pi: \mathbb{R}^{d} \rightarrow \mathbb{R}^{e}$ is a linear projection with $e \leq d$. We may assume that 0 is in the interior of $P$ and thus there are linear forms $\ell_{0}, \ldots, \ell_{m}: \mathbb{R}^{d} \rightarrow \mathbb{R}$ such that

$$
P=\left\{x \in \mathbb{R}^{d}: \ell(x) \leq 1 \text { for all } i=0, \ldots, m\right\} .
$$

For a face $F \subseteq P$, let $I(F)=\left\{i \in[m]: \ell_{i}(x)=1\right.$ for all $\left.x \in F\right\}$, where $[m]=\{0, \ldots, m\}$, and set

$$
\mathcal{I}=\mathcal{I}(P):=\{I(F): F \subseteq P \text { face }\} .
$$

Since $I(F) \neq I\left(F^{\prime}\right)$ if and only if $F \neq F^{\prime}, \mathcal{I}$ ordered by inclusion is isomorphic to (the opposite of) the face lattice of $P$ and we consider this to be the combinatorial information obtained from $P$.

Now, if $\pi: \mathbb{R}^{d} \rightarrow \mathbb{R}^{e}$ is a linear projection, then its adjoint is an injection $\pi^{*}:\left(\mathbb{R}^{e}\right)^{*} \hookrightarrow\left(\mathbb{R}^{d}\right)^{*}$ and let $L \subseteq\left(\mathbb{R}^{d}\right)^{*}$ be its image. Finally, let $\phi:\left(\mathbb{R}^{d}\right)^{*} \rightarrow\left(\mathbb{R}^{d}\right)^{*} / L \cong \mathbb{R}^{d-e}$ be the canonical projection. We define the $P$-transform of $\pi$ as the point configuration $G_{P}:[m] \rightarrow \mathbb{R}^{d-e}$ by $G_{P}(i)=\phi\left(\ell_{i}\right)$ for $i=0, \ldots, m$. This, in a strong way, depends on the geometry of $P$. The following lemma is the analog of Lemma 3.1 that has been discovered in different contexts. See [29, 23] for strengthenings.

Lemma 4.1. Let $P \subset \mathbb{R}^{d}$ be a full-dimensional polytope. For $\pi: \mathbb{R}^{d} \rightarrow \mathbb{R}^{e}$, let $G_{P}$ be the associated $P$-transform. For a proper face $F \subset P$ the following are equivalent

(i) $F^{\prime}=\pi(F)$ is a proper face of $\pi(P)$ and $\pi^{-1}\left(F^{\prime}\right) \cap P=F$,

(ii) $0 \in$ relint conv $G_{P}(I(F))$.

Proof. Let $\ell^{\prime} \in\left(\mathbb{R}^{e}\right)^{*}$ be a linear function such that $\ell^{\prime}$ is maximized on $F^{\prime}$ over $\pi(P)$. This is the case if $\ell=\pi^{*}\left(\ell^{\prime}\right)$ is maximized on $F$ over $P$. That is, if there are scalars $\lambda_{i} \geq 0$ for 
$i \in I(F)$ such that

$$
\ell=\sum_{i \in I(F)} \lambda_{i} \ell_{i}
$$

Since $\ell$ is in the image of $\pi^{*}$, it follows that $\phi(\ell)=0$ and hence, after applying $\phi$ to (4) and scaling by $1 / \sum \lambda_{i}$ we get that $0 \in \operatorname{conv} G_{P}(I(F))$. If there is no face $\widehat{F} \supset F$ such that $\pi(\widehat{F})=F^{\prime}$, then all the $\lambda_{i}$ must be strictly positive, and hence the origin is in the relative interior of conv $G_{P}(I(F))$.

For the converse, note that if there are $\lambda_{i}>0$ for $i \in I(F)$ such that $\ell=\sum_{i \in I(F)} \lambda_{i} \ell_{i}$, then $F$ is the inclusion-maximal face that maximizes $\ell$. Moreover, if $\ell$ is in the kernel of $\phi$, then, by definition, it is in the image of $\pi^{*}$ and hence $\ell=\pi^{*}\left(\ell^{\prime}\right)$ for some $\ell^{\prime} \in\left(\mathbb{R}^{e}\right)^{*}$. That is, $\pi(F)$ maximizes $\ell^{\prime}$ over $\pi(P)$.

The following example shows the connection to usual Gale transforms.

Example 4.2. For $n \geq 1$ fixed, let $\Delta \subset \mathbb{R}^{n-1}$ be the $(n-1)$-dimensional simplex with vertices $v_{1}, v_{1}, \ldots, v_{n} v_{n}=-\left(v_{1}+\cdots+v_{n-1}\right)$. Its $n$ facet-defining linear inequalities $\ell_{i}(x) \leq 1$ satisfy $\ell_{i}\left(v_{j}\right)=1$ if $i \neq j$ and $<1$ otherwise. If we write $F_{J}=\operatorname{conv}\left(v_{i}: i \in J\right)$ for the face corresponding to a subset $J \subseteq[n]$, then the convention above yields $I\left(F_{J}\right)=[n] \backslash J$.

For a given polytope $Q=\operatorname{conv}\left(q_{1}, \ldots, q_{n}\right) \subset \mathbb{R}^{d}$ on $n$ vertices whose barycenter is the origin, there is a canonical surjective linear projection $\pi: \mathbb{R}^{n-1} \rightarrow \mathbb{R}^{d}$ that takes $v_{i}$ to $q_{i}$ for all $1 \leq i \leq$ $n$. The corresponding $\Delta$-transform is then a point configuration $G_{\Delta}:[n] \rightarrow\left(\mathbb{R}^{n-1}\right)^{*} / \operatorname{im}\left(\pi^{*}\right) \cong$ $\mathbb{R}^{n-1-d}$ and Lemma 4.1 yields that for any $J \subseteq[n]$ we have that

$$
\pi\left(F_{J}\right)=\operatorname{conv}\left(q_{i}: i \in J\right) \subseteq Q
$$

is a face of $Q$ if and only if $0 \in \operatorname{relint} \operatorname{conv}\left(G_{\Delta}(i): i \in[n] \backslash J\right)$.

This is exactly the statement of Lemma 3.1. Actually, for a careful choice of the simplex $\Delta$, ordinary Gale transforms are exactly $\Delta$-transforms. In general $P$-transforms are a very powerful tool to study polytopes under projections; cf. [22]. In particular, the (well-)known cs transforms for centrally-symmetric polytopes and zonal transforms for zonotopes are $P$ transforms for crosspolytopes and cubes, respectively [19].

Of course, we may also fix the projection $\pi: \mathbb{R}^{d} \rightarrow \mathbb{R}^{e}$ and vary the polytope $P \subset \mathbb{R}^{d}$. This approach directly allows us to associate a colorful configuration to a Minkowski sum. For fixed $s$ and $d$, the Minkowski projection is the linear map $\mu:\left(\mathbb{R}^{d}\right)^{s+1} \rightarrow \mathbb{R}^{d}$ given by

$$
\left(x_{0}, \ldots, x_{s}\right) \mapsto x_{0}+\cdots+x_{s} .
$$

For polytopes $P_{0}, \ldots, P_{s} \subset \mathbb{R}^{d}$ it is obvious that $\mu\left(P_{0} \times \cdots \times P_{s}\right)=P_{0}+\cdots+P_{d}$. If $P_{i}$ has facetdefining linear forms indexed by $\mathcal{F}_{i}$, then the facet-defining linear forms of $P=P_{0}+\cdots+P_{s}$ are indexed by $\mathcal{F}=\mathcal{F}_{0} \cup \cdots \cup \mathcal{F}_{s}$. The Minkowski transform $M: \mathcal{F} \rightarrow \mathbb{R}^{(s-1) d}$ of $P_{0}, \ldots, P_{s}$ is then the $\left(P_{0} \times \cdots \times P_{s}\right)$-transform for the Minkowski projection $\mu$. This is a colorful configuration. It is also centered, because the normal vectors to the facets of each polytope are positively spanning in their linear span. For faces $F_{j} \subseteq P_{j}, 0 \leq j \leq s$, write $I\left(F_{j}\right) \subseteq \mathcal{F}_{j}$. Then Lemma 4.1 yields that $F_{0}+\cdots+F_{s}$ is a proper face of $P_{0}+\cdots+P_{s}$ if and only if

$$
0 \in \text { relint conv } M\left(I_{0}\left(F_{0}\right) \cup \cdots \cup I_{s}\left(F_{s}\right)\right) .
$$

Note that $M$ is a colorful configuration in $(s+1) d$-dimensional space and thus typically of much lower dimension than the colorful Gale transform of Section 3. However, as we pointed 
out in Section 3, the colorful Gale transform also contains information about subsums $\sum_{i \in I} P_{i}$ for any $I \subseteq[s]$, and in particular, of each of the individual summands. This is not true any more for the Minkowski transform $M$. In general, the number of points in the two transforms does not coincide either: The Minkowski transform is indexed by facets and the colorful Gale transform by vertices.

Yet, these two approaches are strongly related. Consider the case where $P_{i} \subset \mathbb{R}^{d}$ is an $\left(n_{i}-1\right)$-dimensional simplex for all $0 \leq i \leq s$. If $\mathcal{V}_{i}$ and $\mathcal{F}_{i}$ index the facets and the vertices of $P_{i}$, respectively, then $\left|\mathcal{V}_{i}\right|=\left|\mathcal{F}_{i}\right|=n_{i}$ and we can identify both by matching each facet with the only vertex it does not contain. Then, as in Example 4.2, for $J_{i} \subset \mathcal{V}_{i}$ the face $F_{J_{i}}=\operatorname{conv}\left(q_{j}: j \in J_{i}\right)$ belongs to the facets indexed by $I\left(F_{j}\right)=\mathcal{F}_{i} \backslash J_{i}$.

The Minkowski transform $M: \mathcal{F} \rightarrow \mathbb{R}^{n-s-d-1}$, where $n=n_{0}+\cdots+n_{s}$ is a centered colorful configuration, because the normal vectors to the facets of each polytope are positively spanning in their linear span. Lemma 4.1 yields that for any collection $J_{i} \subseteq \mathcal{V}_{i}$ for $0 \leq i \leq s$, the sum $F_{J_{0}}+\cdots+F_{J_{s}}$ is a face of $P_{0}+\cdots+P_{s}$ if and only if

$$
0 \in \operatorname{conv}\left(M\left(\mathcal{F}_{0} \backslash J_{0}\right) \cup \cdots \cup M\left(\mathcal{F}_{s} \backslash J_{s}\right)\right) .
$$

Notice how this is exactly the condition of Proposition 3.4. This shows that the Minkowski transformation $M$ and the colorful Gale transform $G$ of $P_{0}, \ldots, P_{s}$ are both colorful configurations in $(n-s-d-1)$-dimensional space with the same combinatorics. A careful computation shows that these actually coincide.

Proposition 4.3. For any collection of simplices $P_{0}, \ldots, P_{s} \subset \mathbb{R}^{d}$, the Minkowski transformation and the colorful Gale transformation coincide up to a choice of coordinates.

\section{REFERENCES}

[1] K. A. Adiprasito And R. Sanyal, Relative Stanley-Reisner theory and upper bound theorems for Minkowski sums, Publications mathématiques de l'IHÉS, (2016), pp. 1-65. 3, 13

[2] J. L. Arocha, I. Bárány, J. Bracho, R. Fabila, and L. Montejano, Very colorful theorems, Discrete Comput. Geom., 42 (2009), pp. 142-154. 1, 10

[3] I. BÁRÁNY, A generalization of Carathéodory's theorem, Discrete Mathematics, 40 (1982), pp. $141-152$. 1,10

[4] I. BÁRÁNy AND J. MATOUŠEK, Quadratically many colorful simplices, SIAM J. Discrete Math., 21 (2007), pp. 191-198. 2

[5] A. BuöRner, Topological methods, in Handbook of combinatorics, Vol. 1, 2, Elsevier, Amsterdam, 1995, pp. $1819-1872.10$

[6] B. Burton, Minimal triangulations and normal surfaces, PhD thesis, The University of Melbourne, 2003. 3,4

[7] A. Deza, S. Huang, T. Stephen, and T. Terlaky, Colourful simplicial depth, Discrete \& Computational Geometry, 35 (2006), pp. 597-615. 1, 2

[8] A. Deza, F. Meunier, and P. Sarrabezolles, A combinatorial approach to colourful simplicial depth, SIAM J. Discrete Math., 28 (2014), pp. 306-322. 2

[9] A. Deza, T. Stephen, And F. Xie, More colourful simplices, Discrete \& Computational Geometry, 45 (2011), pp. 272-278. 2

[10] P. Gritzmann And B. Sturmfels, Minkowski addition of polytopes: computational complexity and applications to Gröbner bases, SIAM J. Discrete Math., 6 (1993), pp. 246-269. 3

[11] B. Grünbaum, Convex polytopes, vol. 221 of Graduate Texts in Mathematics, Springer-Verlag, New York, second ed., 2003. 12

[12] A. Hatcher, Algebraic topology, Cambridge University Press, Cambridge, 2002. 10

[13] A. Holmsen, J. Pach, And H. Tverberg, Points surrounding the origin., Combinatorica, 28 (2008), pp. 633-644. 1, 10 
[14] B. Huber, J. Rambau, and F. Santos, The Cayley trick, lifting subdivisions and the Bohne-Dress theorem on zonotopal tilings, J. Eur. Math. Soc. (JEMS), 2 (2000), pp. 179-198. 12

[15] R. Y. Liu, On a notion of data depth based on random simplices, Ann. Statist., 18 (1990), pp. 405-414. 1

[16] J. MatoušEK, Lectures on discrete geometry, vol. 212 of Graduate Texts in Mathematics, Springer-Verlag, New York, 2002. 11

[17] — Using the Borsuk-Ulam theorem, Universitext, Springer-Verlag, Berlin, 2003. Lectures on topological methods in combinatorics and geometry, Written in cooperation with Anders Björner and Günter M. Ziegler. 5

[18] B. Matschke, J. Pfeifle, and V. Pilaud, Prodsimplicial-neighborly polytopes, Discrete Comput. Geom., 46 (2011), pp. 100-131. 3

[19] P. McMullen, Transforms, diagrams and representations, in Contributions to Geometry, J. Tölke and J. M. Wills, eds., Birkhäuser Basel, 1979, pp. 92-130. 4, 14, 15

[20] F. Meunier And A. Deza, A further generalization of the colourful Carathéodory theorem, in Discrete geometry and optimization. Selected papers based on the presentations at the conference and workshop, Toronto, Canada, September 19-23, 2011, vol. 69 of Fields Institute Communications, Springer, New York, 2013, pp. 179-190. 1, 10

[21] B. Nill And A. PAdrol, The degree of point configurations: Ehrhart theory, Tverberg points and almost neighborly polytopes, European Journal of Combinatorics, 50 (2015), pp. 159 - 179. 12

[22] T. RöRig and R. Sanyal, Non-projectability of polytope skeleta, Adv. Math., 229 (2012), pp. 79-101. 3, 15

[23] R. Sanyal and G. M. Ziegler, Construction and Analysis of Projected Deformed Products., Discrete \& Computational Geometry, 43 (2010), pp. 412-435. 14

[24] P. Sarrabezolles, The colourful simplicial depth conjecture, Journal of Combinatorial Theory, Series A, 130 (2015), pp. $119-128.2$

[25] R. Schneider, Convex bodies: the Brunn-Minkowski theory, vol. 151 of Encyclopedia of Mathematics and its Applications, Cambridge University Press, Cambridge, expanded ed., 2014. 3

[26] T. Stephen and H. Thomas, A quadratic lower bound for colourful simplicial depth, Journal of Combinatorial Optimization, 16 (2008), pp. 324-327. 2

[27] G. WeGner, d-collapsing and nerves of families of convex sets, Arch. Math. (Basel), 26 (1975), pp. 317321. 10

[28] G. M. Ziegler, Lectures on Polytopes., vol. 152 of Graduate Texts in Mathematics, Springer, New York, 1995. 11, 13

[29] G. M. Ziegler, Projected products of polygons, Electron. Res. Announc. Amer. Math. Soc., 10 (2004), pp. 122-134 (electronic). 14

Einstein Institute for Mathematics, Hebrew University of Jerusalem, Jerusalem, Israel

E-mail address: adiprasito@math.huji.ac.il

Fachbereich Mathematik und Informatik, Freie Universität Berlin, Berlin, Germany

E-mail address: pbrinkmann@math.fu-berlin.de

Sorbonne Universités, Université Pierre et Marie Curie (Paris 6), Institut de Mathématiques De Jussieu - Paris Rive Gauche (UMr 7586), Paris, France

E-mail address: arnau.padrol@imj-prg.fr

Einstein Institute for Mathematics, Hebrew University of Jerusalem, Jerusalem, Israel

E-mail address: ppatak@seznam.cz

Einstein Institute for Mathematics, Hebrew University of Jerusalem, Jerusalem, Israel

E-mail address: zuzka@kam.mff.cuni.cz

Fachbereich Mathematik und Informatik, Freie Universität Berlin, Berlin, Germany

E-mail address: sanyal@math.fu-berlin.de 